\title{
INVOLUTORY AUTOMORPHISMS OF GROUPS OF ODD ORDER
}

\author{
J. N. WARD 1 \\ (Received 3 December 1965)
}

\section{Introduction}

Let $G$ be a finite group of odd order with an automorphism $\omega$ of order 2 . The Feit-Thompson theorem implies that $G$ is soluble and this is assumed throughout the paper. Let $G_{\omega}$ denote the subgroup of $G$ consisting of those elements fixed by $\omega$. If $F(G)$ denotes the Fitting subgroup of $G$ then the upper Fitting series of $G$ is defined by $F_{1}(G)=F(G)$ and $F_{r+1}(G)=$ the inverse image in $G$ of $F\left(G / F_{r}(G)\right) . G^{(r)}$ denotes the rth derived group of $G$. The principal result of this paper may now be stated as follows:

THEOREM 1. Let $G$ be a group of odd order with an automorphism $\omega$ of order 2. Suppose that $G_{\omega}$ is nilpotent, and that $G_{\omega}^{(r)}=1$. Then $G^{(r)}$ is nilpotent and $G=F_{3}(G)$.

Examples given in [7] show that there exist groups $G$ satisfying the hypothesis of theorem 1 for which $G \neq F_{2}(G)$. If $H$ is any nilpotent group of odd order and derived length $r$, we can construct a group $G$ satisfying the hypothesis of the theorem such that $G_{\omega} \cong H$ and $G^{(r-1)}$ is not nilpotent. Indeed let $q$ be an odd prime not dividing the order of $H$ and construct the group algebra $A$ of $K$, the direct product of $H$ and the cyclic group of order 2, over $G F(q)$, the Galois field with $q$ elements. The mappings

$$
x \rightarrow a x+b
$$

of $A$ into itself, where a runs over $K$ and $b$ runs over $A$, form a group $\Gamma$. $\Gamma$ has a subgroup $G$ of odd order and index $2, G / F(G) \cong H$ and an inner automorphism of $\Gamma$ of order 2 induces an automorphism $\omega$ of $G$ with $G_{\omega} \cong H$.

L. Kovacs and G. E. Wall have constructed in [7] $p$ groups of arbitrarily high derived length, each with an automorphism $\omega$ of order 2 such that the fixed point group of $\omega$ is cyclic. Taking $K$ to be the splitting extension of a suitable one of these groups by its automorphism and applying the above construction we can show that given any integer $n$ there exists a group $G$ of odd

1 Work on this problem was partly completed whilst the author held a C.S.I.R.O. studentship. 
order with an automorphism $\omega$ of order 2 such that $G_{\omega}$ is metabelian and $G^{(n)}$ is not nilpotent. Thus the assumption that $G_{\omega}$ is nilpotent in theorem 1 is essential.

If the group $G$ has several automorphisms of order 2 satisfying the condition that each of the fixed point groups is nilpotent, then stronger assertions can be made. We have

THEOREM 2. Let $G$ be a group of odd order with a group of automorphisms $A$ of order 4 and exponent 2 such that for each $\omega \in A, \omega \neq 1, G_{\omega}$ is nilpotent. Then $G^{\prime}$ is nilpotent.

Under these conditions $G$ need not be nilpotent but with even stronger hypotheses the nilpotence of $G$ can be asserted:

THEOREM 3. Let $G$ be a group of odd order with a group of automorphisms $A$ of order 8 and exponent 2 such that for each $\omega \in A, \omega \neq 1, G_{\omega}$ is nilpotent. Then $G$ is nilpotent.

A very much more elementary result is

THEOREM 4. Let $G$ be a group of odd order with an automorphism $\omega$ of order 2 . If $G_{\omega}$ is a Hall-subgroup of $G$ then there exists a normal abelian complement of $G_{\omega}$ in $G$.

For further discussion of theorems of this kind we refer to [7].

I wish to express my thanks to G. E. Wall for his guidance in this work.

Notation. The notation is standard and agrees with that mentioned in [7]. By a proper subgroup is means a subgroup not equal to the whole group. A non-trivial subgroup is one containing more than one element. If $G$ is a group, $|G|$ denotes the order of $G, Z(G)$ the centre of $G$ and $\Phi(G)$ the Frattini subgroup of $G$. For subgroups $H$ and $K$ of $G,|G: H|$ is the index of $H$ in $G, C_{H}(K)$ the centralizer of $K$ in $H$ and $N_{H}(K)$ the normalizer of $K$ in $H$.

$\mathscr{F}$ always denotes the algebraic closure of $G F(p)$, the Galois field with $p$-elements. If $\mathscr{L}$ is a field $\mathscr{L}(G)$ denotes the group algebra of $G$ over $\mathscr{L}$. If $V$ is an $\mathscr{L}(G)$-module, we write scalars as left operators on $V$ and elements of $\mathscr{L}(G)$ as right operators on $V$.

If $p$ is a prime, a $p^{\prime}$ group is a group of order prime to $p$. A Hall $p^{\prime}$ subgroup of a group is a Hall subgroup, whose index is a power of $p$.

A frequently used property of a soluble group $G$ is that $C_{G}(F(G)) \leqq F(G)$ ([1], p. 646).

\section{Preliminary lemmas}

Lemma 1. Let $P$ be a p-group and $H$ a proper subgroup of $P$. Then $|P: H|>\left|P^{\prime}: H \cap P^{\prime}\right|$. 
Proof. Since $P^{\prime} \leqq \Phi(P),\left|P: P^{\prime}\right|>\left|P^{\prime} H: P^{\prime}\right|$. The result follows.

LEMMA 2. Let $G$ be a soluble group operated on by a group $A$ of automorphisms. Suppose that for some pair of integers $\left.(m, n),(n>0), G^{(m)} \$ F_{n} G\right)$ but if $H / K$ is any $A$-section ${ }^{2}$ of $G, H / K \neq G / 1$, then $(H / K)^{(m)} \leqq F_{n}(H / K)$.

Then if $H$ is a non trivial normal $A$-subgroup of $G, F(G) \leqq H . F(G)$ is an elementary abelian $p$-group for some prime $p$.

Proof. It follows from the hypothesis that if $1 \neq H, K \neq 1$ are norma] $A$-subgroups of $G$ then $\mathrm{I} \neq H \cap K$. Thus $G$ has a unique minimal normal $A$. subgroup $M$. Since $G$ is soluble, $M$ is an elementary abelian $p$-group. Now from [1] p. 647, $F(G / \Phi(G))=F(G) / \Phi(G)$ so $F_{n}(G / \Phi(G))=F_{n}(G) / \Phi(G)$ Thus $\Phi(G)=1$. Since $\Phi(N) \leqq \Phi(G)$ if $N \triangleleft G$ ([3], p. 162), $F(G)$ is ar elementary abelian $p$-group.

Write $H / M=F(G / M)$. Then $F(G)$ is the Sylow $p$-subgroup of $H$ Since $(G / M)^{(m)} \leqq F_{n}(G / M)$ whilst $G^{(m)} \leqq F_{n}(G), H$ properly contain! $F(G)$.

As $F(G)$ is an elementary abelian normal Sylow $p$-subgroup of $H, F(G$. is a completely reducible $H / F(G)$ module. Thus $F(G)=M \times N$ wher $N \triangleleft H$. Since $H / M$ is nilpotent and $F(G)$ is abelian, $N \leqq Z(H)$. Suppose $Z(H)>1$. Then $Z(H)$ is characteristic in the normal $A$-subgroup $H$ of $G$ so $Z(H)$ is a normal $A$-subgroup of $G$. Hence $Z(H) \geqq F(G)$ so $H \leqq C_{G}(F(G)$ '. $=F(G)$, a contradiction. Thus $N \leqq Z(H)=1$ and $F(G)=M$, provin the lemma.

We apply lemma 2 in the following way. Each of theorems 1, 2 and: is to be proved by induction on the order of $G$ and by way of contradiction For theorem 1 take $A$ to be the group $\{1, \omega\}$. Let $G$ be a group of minima order not satisfying the hypothesis of the theorem in question. For theorem we take $(m, n)=(0,3)$ or $(r, 1)$; for theorem $2,(m, n)=(1,1)$ and for theoren 3, $(m, n)=(0,1)$. Now if $H / K \neq G / 1$ is an $A$-section of $G$, either $A$ is rep resented faithfully as a group of automorphisms of $H / K$ in which cas by induction $(H / K)^{(m)} \leqq F_{n}(H / K)$ or for some automorphism $\omega \in A$ $(H / K)_{\omega}=H / K$ so $H / K$ is nilpotent being isomorphic to a section of $G_{\omega}$ Thus in either case since $|G|$ is odd the hypothesis of the lemma is satisfie and we conclude that $F(G)$ is the unique minimal normal $A$-subgroup of $G$.

The following lemma and its corollaries are stated for convenience. Th method of proof is well known, see for example [7].

LEMMA 3. Let $G$ be a group of odd order with an automorphism $\omega c$ order 2. Then there exists precisely one element of $G$ which is inverted by c in each left (right) coset of $G_{\omega}$. of $G$.

2 An $A$-section of $G$ is a factor group $H / K$ where $K \triangleleft H$ and $H$ and $K$ are $A$-subgrouf 
COROLLARY 1. Let $G$ be a group of odd order with an automorphism $\omega$ of order 2. Every element of $G$ may be expressed as the product of an element fixed by $\omega$ and an element inverted by $\omega$.

COROLLARY 2. Let $G$ be a group of odd order with an automorphism $\omega$ of order 2. Let $H$ be a subgroup of $G$ containing $G_{\omega}$. Then $H^{\omega}=H$.

COROLLARY 3. Let $G$ be an abelian group of odd order with an automorphism $\omega$ of order 2. Then if $N$ is the set of elements of $G$ which are inverted by $\omega, N$ is a subgroup of $G$ and $G=N \times G_{\omega}$.

Theorem 4 follows from lemma 3 :

The Hall-subgroups of $G$ which complement $G_{\omega}$ form a characteristic system of subgroups. Since $G$ is of odd order one of these is fixed by $\omega$; this subgroup consists of those elements of $G$ inverted by $\omega$ and so is normalized by $G_{\omega}$. Thus it is a normal abelian complement of $G_{\omega}$ in $G$.

\section{Proof of theorem 1}

The theorem is proved by induction on $|G|$ and by way of contradiction. Suppose therefore that $G$ is a group of minimal order satisfying the hypothesis of the theorem but not the conclusion. It follows from [7] that the theorem is true for $r=1$ so we may assume $r>1$. Since $|G|$ is odd, $G$ is soluble. We have already proved.

LEMMA 1. $F(G)$ is the unique minimal normal $\omega$-subgroup of $G$. Therefore $F(G)$ is an elementary abelian $p$-group for some prime $p$.

Notation. For each positive integer $n$, set $F_{n}=F_{n}(G)$. Let $\Gamma$ denote the splitting extension of $G$ by $\omega$ and $p$ the unique prime dividing $\left|F_{1}\right|$.

LEMMA 2. (i) $\left(G / F_{1}\right)_{\omega} \neq G / F_{1}$,

(ii) $F_{1}$ is a faithful irreducible $\Gamma / F_{1}$-module,

(iii) $\left(F_{1}\right)_{\omega}>1$. Therefore $p \| G_{\omega} \mid$.

Proof. (i) If $\left(G / F_{1}\right)_{\omega}=G / F_{1}$, then $G / F_{1}$ is isomorphic to a section of $G_{\omega}$ and therefore is nilpotent of derived length less than or equal to $r$. It follows that $G$ satisfies the conclusion of the theorem. Hence $\left(G / F_{1}\right)_{\omega} \neq$ $G / F_{1}$.

(ii) Lemma 1 implies that $F_{1}$ is an irreducible $\Gamma / F_{1}$-module. To prove that $F_{1}$ is a faithful $\Gamma / F_{1}$-module we need to prove that $C_{\Gamma}\left(F_{1}\right)=F_{1}$. Since $F_{1}$ is the Fitting subgroup of $G$, and since $G$ is soluble, $C_{G}\left(F_{1}\right)=F_{1}$. Hence if $C_{\Gamma}\left(F_{1}\right)>F_{1},\left|C_{\Gamma}\left(F_{1}\right): F_{1}\right|=2$. In this case $\Gamma / F_{1}$ has a normal Sylow 2-subgroup so that $\Gamma / F_{1}=G / F_{1} \times g p\left\{\omega F_{1}\right\}$ from which it follows that $\left(G / F_{1}\right)_{\omega}=G / F_{1}$, contradicting (i). This proves (ii). 
(iii) $\left(F_{1}\right)_{\omega}>1$ for if $\left(F_{1}\right)_{\omega}=1, \omega$ must invert all the elements of $F_{1}$. Then, since $\Gamma / F_{1}$ is faithfully represented by its action on $F_{1}, \omega F_{1}$ lies in the centre of $\Gamma / F_{1}$. But this again implies that $\left(G / F_{1}\right)_{\omega}=G / F_{1}$, contradicting (i).

Lemma 3. $F_{2} / F_{1}$ is a $p^{\prime}$-group. $G / F_{1}$ has no non-trivial normal $p$-subgroups.

Proof. Suppose that $P / F_{1}$ is the Sylow $p$-subgroup of $F_{2} / F_{1}$. Then as $F_{1}$ is a $p$-group, $P$ is a normal $p$-subgroup of $G$. Hence $P \leqq F_{1}$. The second statement follows from the first.

Lemma 4. If $G_{\omega}$ is a p-group then $G=F_{2} G_{\omega}$ and $\left(G_{\omega}\right)^{(r-1)}$ is not contained in $F_{1} . F_{2} / F_{1}$ is abelian.

Proof. We know, by lemma 2 , that $F_{1}$ is a $p$-group and, by lemma 3, that $F_{2} / F_{1}$ is a $p^{\prime}$-group. Since $G / F_{1}$ is soluble and $F_{2} / F_{1}$ is a normal subgroup of $G / F_{1}, F_{2} / F_{1}$ is contained in every Hall $p^{\prime}$-subgroup of $G / F_{1}$. Now the Hall $p^{\prime}$-subgroups of $G / F_{1}$ are all conjugate and the order of $G$ is odd so the number of Hall $p^{\prime}$-subgroups is odd. Clearly the automorphism $\omega$ permutes these Hall $p^{\prime}$-subgroups and since the number of them is odd, at least one is fixed by $\omega$. Thus we can choose a Hall $p^{\prime}$-subgroup $H / F_{1}$ such that $H^{\omega}=H$. Now $H_{\omega}=H \cap G_{\omega}$ is a $p$-group so that $H_{\omega} \leqq F_{1}$. Thus $\omega$ acts as a regular automorphism on $H / F_{1}$ so that $H / F_{1}$ is abelian. Since $G_{/} F_{1}$ is a soluble group, $C_{G / F_{1}}\left(F_{2} / F_{1}\right) \leqq F_{2} / F_{1}$. But $F_{2} / F_{1} \leqq H / F_{1}$ so that as $H / F_{1}$ is abelian, $H / F_{1} \leqq C_{G / F_{1}}\left(F_{2} / F_{1}\right) \leqq F_{2} / F_{1} \leqq H / F_{1}$. Thus $F_{2} / F_{1}$ is the unique Hall $p$ 'subgroup of $G / F_{1}$. It follows that $G / F_{2}$ is a $p$-group. Therefore $G=F_{3}$.

Since $G=F_{3}$ and $G$ does not satisfy the conclusion of the theorem, $G^{(r)}$ is not nilpotent.

Suppose by way of contradiction that $G_{\omega}^{(r-1)} \leqq F_{1}$. Then $\left(G / F_{1}\right)_{\omega}$ has derived length at most $r-1$, so by the minimality of $G,\left(G / F_{1}\right)^{(r-1)}$ is nilpotent. Thus $G^{(r-1)} \leqq F_{2}$ and since, as we have already seen, $F_{2} / F_{1}$ is abelian, $G^{(r)} \leqq F_{1}$. This contradiction proves that $G_{\omega}^{(r-1)}$ is not contained in $F_{1}$.

Finally we show that if $G_{\omega} F_{2}<G, G_{\omega}^{(r-1)}$ is contained in $F_{1}$. It then follows from the conclusion of the previous paragraph that $G_{\omega} F_{2}=G$. Suppose then that $G_{\omega} F_{2}<G$, and let $K$ be a maximal subgroup of $G$ containing $G_{\omega} F_{2}$. Since $K$ is a maximal subgroup of $G$ containing $F_{2}$ anc since $G / F_{2}$ is nilpotent, $K$ is a normal subgroup of $G$. By $\S 2$, lemma 3 corollary 2 , as $G_{\omega} \leqq G_{\omega} F_{2} \leqq K, K$ is a $\omega$-subgroup of $G$. Therefore, by the minimality of $G, K^{(r)}$ is nilpotent. But $K^{(r)}$ is a characteristic subgroup o $K$, a normal subgroup of $G$, and therefore $K^{(r)}$ is a normal subgroup of $G$ Hence $K^{(r)} \leqq F_{1}$ so that $K^{(r-1)} \leqq F_{2}$. Now $G_{\omega}^{(r-1)} \leqq K^{(r-1)} \leqq F_{2}$. But $G_{\omega}$ i: 
a $p$-group and $F_{1}$ is the Sylow $p$-subgroup of $F_{2}$ so $G_{\omega}^{(r-1)} \leqq F_{1}$. This completes the proof of the lemma.

We have shown that $F_{1}$ is the unique minimal normal $\omega$-subgroup of $G$. Since $G$ is a normal subgroup of $\Gamma, F(G) \leqq F(\Gamma)$. If $F(\Gamma) \neq F(G)$ then $|F(\Gamma): F(G)|=2$ so that $\omega \in F(\Gamma)$. But in this case, since $F(\Gamma)$ is nilpotent, $\left(F_{1}\right)_{\omega}=F_{1}$ contradicting lemma $2(i i)$. Thus $F_{1}=F(\Gamma)$ is the unique minimal normal subgroup of $\Gamma .|\Gamma: G|=2$ so the solubility of $\Gamma$ follows from that of $G$. Therefore ([1], p. 651) there exists a complement $N$ of $F_{1}$ in $\Gamma$. By Sylow's theorem we can suppose, by taking a suitable conjugate of $N$ if necessary, that $\omega \in N$. Let $M=G \cap N$. Then $M$ is a complement of $F_{1}$ in $G$.

Since the elements of $N$ form a complete set of coset representatives of $F_{1}$ in $\Gamma$, we may consider $F_{1}$ as a $G F(p)(N)$-module. We now summarize the results obtained so far in module notation.

(1) $F_{1}$ is a faithful irreducible $N$-module over $G F(p)$.

(2) $\left(F_{1}\right)_{\omega}>0$.

(3) If $f \in\left(F_{1}\right)_{\omega}$ and $x_{i} \in\left(M_{\omega}\right)^{(i)} \quad(i=0, \cdots, r-1)$ then $f\left(1-x_{0}\right)\left(1-x_{1}\right) \cdots\left(1-x_{r-1}\right)=0$.

(4) If $f \in\left(F_{1}\right)_{\omega}$ and $x \in M_{\omega}$ is of order prime to $p$, then since $G_{\omega}$ is nilpotent, $f x=f$.

It also follows from lemma 2 that $M_{\omega} \neq M$.

If we extend the field of scalars from the prime field $G F(p)$ to its algebraic closure $\mathscr{F}, F_{1}$ splits into a direct sum of absolutely irreducible $\mathscr{F}(N)$ modules, which are algebraically conjugate. ([2], section 70). Taking $V$ as one of these irreducible $\mathscr{F}(N)$-modules, we obtain an $\mathscr{F}(N)$ module with the following properties:

(1) $V_{1}$ is a faithful irreducible $N$-module over $\mathscr{F}$,

(2) $V_{\omega}=\{v \in V \mid v \omega=v\}>0$,

(3) If $v \in V_{\omega}$ and $x_{i} \in\left(M_{\omega}\right)^{(i)} \quad(i=0,1, \cdots, r-1)$ then $v\left(1-x_{0}\right)\left(1-x_{1}\right) \cdots\left(1-x_{r+1}\right)=0$,

(4) If $v \in V_{\omega}$ and $x \in M_{\omega}$ is of order prime to $p$, then $v x=v$.

Notation. $Q=F(M)$.

Lemma 5, $V$ is an irreducible $\mathscr{F}(M)$-module.

Proof. By way of contradiction suppose that there exists an irreducible $\mathscr{F}(M)$-submodule $W$ of $V$ such that $0<W<V$. Since $W \omega$ is also an irreducible $\mathscr{F}(M)$-submodule of $V$ and since $W+W \omega$ is an $\mathscr{F}(N)$-module we have $V=W+W \omega$ and so as an $\mathscr{F}(M)$-module

$$
V=W+W \omega \text {. }
$$


Suppose that $G_{\omega}$ is not a $p$-group. Then there exist an element $x \neq 1$ in $M_{\omega}$ of order prime to $p$. Let $w \in W$ be arbitrary. Then $w+w \omega \in V_{\omega}$ so by property (4) of $V$, $(w+w \omega) x=w+w \omega$. Equating the $W$ and $W \omega$ components of both sides we deduce that $x$ acts trivially on both $W$ and $W \omega$ and so on $V$. But this contradicts property (1) of $V$. Hence we may assume that $G_{\omega}$ is a $p$-group.

Let $x \in Q$ and suppose that for all $w \in W, w x=w$. Since $x \in Q$ and $G_{\omega}$ is a $p$-group, $x^{\omega}=x^{-1}$. Thus if $w \in W, w \omega x=w x^{-1} \omega=w \omega$ so $x$ also acts trivially on $W \omega$. Hence $x$ acts trivially on $W+W \omega=V$ so $x=1$. Therefore $W$ is a faithful $Q$-module. Since $Q=F(M)$ and $M$ is soluble, any normal subgroup of $M$ has non-trivial intersection with $Q$. Hence if $W$ were not a faithful $M$-module, $W$ would not be a faithful $Q$-module. It follows that $W$ is a faithful $M$-module.

Let $w \in W$. Then $w+w \omega \in V_{\omega}$ so if $x_{i} \in\left(M_{\omega}\right)^{(i)}(i=0,1, \cdots, r-1)$ it follows from property (3) of $V$ that

$$
(w+w \omega)\left(1-x_{0}\right)\left(1-x_{1}\right) \cdots\left(1-x_{r-1}\right)=0
$$

and hence equating the $W$-components we have

$$
w\left(1-x_{0}\right)\left(1-x_{1}\right) \cdots\left(1-x_{r-1}\right)=0 .
$$

Consider $W$ as an $\mathscr{F}(Q)$-module. Since $W$ is an irreducible $\mathscr{F}(M)$-module it follows that

$$
W=W_{1}+\cdots W_{n}
$$

where $W_{1}, W_{2}, \cdots, W_{n}$ are the homogeneous components of $W$ as an $\mathscr{F}(Q)$-module. ([2], section 49$)$. Since $M_{\omega}$ is a $p$-group, $Q$ is an abelian $p^{\prime}$ group by lemma 4 . Thus as $\mathscr{F}$ is of characteristic $p$ and algebraically closed, the irreducible $\mathscr{F}(Q)$-submodules of $W$ are one-dimensional. Thus the action of $x \in Q$ on $w \in W_{i}$ may be described by

$$
w x=\chi_{i}(x) w .
$$

$M / Q$ is a transitive permutation group on the $W_{i}$. Since, by lemma 4, $M_{\omega}$ is a complement of $Q$ in $M$, we may consider $M_{\omega}$ as a transitive permutation group on the $W_{i}$. Set $H_{i}=\left\{x \in M_{\omega} \mid W_{i} x=W_{i}\right\}$.

We now prove that if $K \leqq H_{1}$ and $K \triangleleft M_{\omega}$ then $K=1$. For all the $H_{i}$ are conjugate in $M_{\omega}$ so $K \leqq H_{i}$ for all $i$. Now let $y \in K, x \in Q$. Then if $w \in W_{i}, w y^{-1} \in W_{i}$ so

$$
w\left(y^{-1} x y\right)=\left(\left(w y^{-1}\right) x\right) y=\chi_{i}(x) w y^{-1} y=w x
$$

Hence $w\left(y^{-1} x y\right)=w x$ for all $w \in W_{i}$ and $x \in Q$. But since $i$ was arbitrary and $W$ is a direct sum of the $W_{i}, y^{-1} x y$ acts on $W$ in the same way as $x$. But $W$ is a faithful $M$-module so $y^{-1} x y=x$, or as $x$ was arbitrary in $Q, y \in C_{M}(Q)$. 
But as $Q=F(M)$ and $M$ is soluble, $C_{M}(Q) \leqq Q$ so $y \in Q \cap M_{\omega}=1$. This proves the statement made at the beginning of the paragraph.

Let $x \in\left(M_{\omega}\right)^{(r)}$ and $w \in W_{1}$. Since $M_{\omega}$ is a $p$-group and $H_{1}$ is a subgroup of $M_{\omega}$ containing no non-trivial normal subgroup of $M_{\omega}$, it follows from $\S 2$, lemma 1 , that

$$
\left|\left(M_{\omega}\right)^{(i)}: M_{\omega}^{(i)} \cap H_{1}\right|>\left|\left(M_{\omega}\right)^{(i+1)}:\left(M_{\omega}\right)^{(i+1)} \cap H_{1}\right|
$$

for all $i$ such that $\left(M_{\omega}\right)^{(i)} \neq 1$. By lemma $4,\left(M_{\omega}\right)^{(r-1)} \neq 1$, so that $\left(^{*}\right)$ is true for $0 \leqq i \leqq r-1$. But $\left|\left(M_{\omega}\right)^{(i)}:\left(M_{\omega}\right)^{(i)} \cap H_{1}\right|$ is the number of $W_{i}$ in the same system of transitivity as $W_{1}$ under $\left(M_{\omega}\right)^{(i)}$. Hence for each $(0 \leqq i \leqq r-1)$ we can choose $x_{i} \in\left(M_{\omega}\right)^{(i)}$ such that $W_{1} x_{i}$ is not in the same system of transitivity as $W_{1}$ under $\left(M_{\omega}\right)^{(i+1)}$. Now

$$
w\left(1-x_{0}\right)\left(1-x_{1}\right) \cdots\left(1-x_{r-1}\right)(1-x)=0 .
$$

Since $W_{1} x_{0}$ is not in the same system of transitivity as $W_{1}$ under $\left(M_{\omega}\right)^{\prime}$ we can conclude that

$$
w\left(1-x_{1}\right) \cdots\left(1-x_{r-1}\right)(1-x)=0
$$

and finally $w(1-x)=0$. Hence $x \in H_{1}$ since $w$ was arbitrary in $W_{1}$. But $x$ was arbitrary in $\left(M_{\omega}\right)^{\langle(r)}$ so that $\left(M_{\omega}\right)^{(r)} \leqq H_{1}$. But $\left(M_{\omega}\right)^{(r)} \triangleleft M_{\omega}$ so $\left(M_{\omega}\right)^{(r)}=1$ contradicting lemma 4 . This completes the proof of lemma 5 .

Lemma 6. If $L \neq 1$ is a normal $\omega$-subgroup of $M$, then $L_{\omega}$ is a nontrivial proper subgroup of $L$.

PROOF. Since $M$ is soluble and every soluble group contains a characteristic subgroup which is abelian, it is sufficient to prove the lemma for abelian $L$. Therefore $L$ is supposed to be a normal abelian subgroup of $M$. Now $L$ is contained in $F(M)=Q$. It follows from lemma 3 that $L$ is a $p^{\prime}$ group. Write

$$
V=V_{1} \oplus V_{2} \oplus \cdots \oplus V_{s}
$$

where $V$ is considered as an $\mathscr{F}(L)$-module and the $V_{i}$ are the homogeneous components. Since $L$ is an abelian $p^{\prime}$-group whilst $\mathscr{F}$ is algebraically closed of characteristic $p$, the action of $x \in L$ on $v \in V_{i}$ may be described by

$$
v x=\chi_{i}(x) v \text {. }
$$

The characters $\chi_{i}$ are all conjugate and the number, $s$, of homogeneous zomponents divides the order of $M([2]$, section 49$)$. Thus none of the characters $\chi_{i}(i=1,2, \cdots, s)$ is the trivial character since $V$ is a faithful module. Also $s$ is odd.

We complete the proof of the lemma by showing that if $L_{\omega}=1$ or $L_{\omega}=L$ then we can choose an $i$ such that $\chi_{i}$ is the trivial character.

Since $\omega$ has order 2 and $V$ is an $\mathscr{F}(N)$-module, for each $i(i=1,2, \cdots, s)$ 
there exists $j$ such that $V_{i} \omega=V_{j}$ and $V_{j} \omega=V_{i}$. Since $s$ is odd there exists at least one $i$ for which $V_{i} \omega=V_{i}$. Suppose $v \in V_{i}$ and $x \in L$. Then $\chi_{i}(x) v_{i} \omega=v_{i} \omega x=v_{i} x^{\omega} \omega=\chi_{i}\left(x^{\omega}\right) v_{i} \omega$ so that $\chi_{i}(x)=\chi_{i}\left(x^{\omega}\right)$, for all $x \in L$. Now if $L_{\omega}=1$, then for all $x \in L, x^{\omega}=x^{-1}$ so that $\chi_{i}(x)=\chi_{i}\left(x^{-1}\right)$ or $\chi_{i}\left(x^{2}\right)=1$. Since $L$ has odd order, it follows that $\chi_{i}$ is the trivial character. Thus $L_{\omega}>1$.

Now suppose that $L_{\omega}=L$. By the second property of $V, V_{\omega}>0$ so that there exists $0 \neq v=v \omega \in V$. Since $L=L_{\omega}$ is a $p^{\prime}$-group, it follows from property (4) of $V$ that $v=v x$ for all $x \in L$. Thus $\{k v \mid k \in \mathscr{F}\}$ is a trivial $\mathscr{F}(L)$ submodule of $V$ and therefore is contained in some $V_{j}$. For this $V_{j}, \chi_{j}=1$ clearly. This contradiction proves the lemma.

Remark. In lemma 6, $L_{\omega}$ cannot be a normal subgroup of $M$, for if it were we would obtain a contradiction by applying lemma 6 to $L_{\omega}$. But $(Z(M))_{\omega}$ is a normal subgroup of $M$ so $Z(M)=1$. Therefore we can now assume that $Q=F(M)$ is a proper subgroup of $M$.

Lемма 7, $Q$ is abelian.

Proof. We consider $V$ as a $\mathscr{F}(Q)$-module and write

$$
V=V_{1} \oplus V_{2} \oplus \cdots \oplus V_{s}
$$

where the $V_{i}$ are the homogeneous components of $V$. Let $Q_{i}$ be the kernel of the representation of $Q$ obtained on $V_{i}$ for each $i=1, \ldots, s$. Then the $Q_{i}$ are all conjugate, ([2], section 49), so that if $Q^{\prime} \leqq Q_{i}$ for some $i$ then $Q^{\prime} \leqq Q_{i}$ for all $i$. Therefore in this case $Q^{\prime}$ is contained in the kernel of $V_{1}+V_{2}+\cdots$ $+V_{s}=V$. But $V$ is a faithful $M$-module so that this implies that $Q^{\prime}=1$, and proves the lemma.

Now suppose that $Q_{\omega}$ is contained in one of the groups $Q_{i}(i=1, \ldots, s)$, say $Q_{j}$. Then by $\S 2$, lemma 3 , corollary $2, Q_{j}^{\omega}=Q_{j}$. Therefore $\omega$ induces a regular automorphism on $Q / Q_{j}$ so that $Q / Q_{j}$ is abelian. Consequently $Q^{\prime} \leqq Q_{j}$. Thus it is sufficient to prove that for some $i, Q_{\omega}$ is contained in $Q_{i}$.

Suppose that there exists an $i$ such that $V_{i} \omega \neq V_{i}$. Let $v \in V_{i}$. Then $v+v \omega \in V_{\omega}$ so that as $Q_{\omega}$ is a $p^{\prime}$-group if $x \in Q_{\omega}$, by the fourth property of $V,(v+v \omega) x=v+v \omega$. Equating the $V_{i}$ components of both sides, we see that $v x=v$ so that $Q_{\omega}$ is contained in $Q_{i}$.

Finally suppose by way of contradiction that $V_{i} \omega=V_{i}$ for all $i$ and fix $i$. Considering $V_{i}$ as a $Z(Q)$-module, we may write

$$
V_{i}=W_{i 1} \oplus W_{i 2} \oplus \cdots \oplus W_{i u}
$$

where for each $j, W_{i j}$ is a homogeneous component of $V_{i}$. Since $V_{i} \omega=V_{i}$ we find, as we have done previously in similar circumstances, that there exists a $j$ such that

$$
W_{i j} \omega=W_{i j}
$$


Since $Z(Q)$ is an abelian $p^{\prime}$-group, the elements of $Z(Q)$ act as scalar multipliers on the $W_{i j}$. Suppose that if $x \in Z(Q)$ and $w \in W_{i j}, w x=\chi_{i j}(x) w$. Then $\chi_{i j}(x) w \omega=w \omega x=w x^{\omega} \omega=\chi_{i j}\left(x^{\omega}\right) w \omega$ so that $\chi_{i j}(x)=\chi_{i j}\left(x^{\omega}\right)$. Since $Z(Q)$ is a non-trivial normal abelian subgroup of $M,(Z(Q))_{\omega}<Z(Q)$ by lemma 6 . Therefore the set $H$ of elements of $Z(Q)$ inverted by $\omega$ forms a nontrivial subgroup of $Z(Q)(\S 2$, lemma 3 , corollary 3$)$. Since $H$ is a subgroup of $Z(Q), H$ is normal in $Q$. Now if $x \in H, \chi_{i j}(x)=\chi_{i j}\left(x^{\omega}\right)=\chi_{i j}\left(x^{-1}\right)$. Since $H$ is of odd order, for all $x \in H, \chi_{i j}(x)=1$. Thus $H$ is contained in the kernel of the representation of $Z(Q)$ given by $W_{i j}$. Since for $k \neq j$, the kernel of $W_{i k}$ is conjugate to that of $W_{i j}$ in $Q$ and since $H$ is a normal subgroup of $Q$, $H$ is contained in the kernel of $W_{i k}$ for all $k$. Thus $H$ is contained in the kernel of $W_{i 1}+\cdots+W_{i u}=V_{i}$. But this is true for all $i$ so that $H$ is contained in the kernel of $V_{1}+V_{2}+\cdots+V_{s}=V$. Since $V$ is a faithful $M$-module, this implies that $H=1$ and this contradiction, to the fact that $H$ is a non-trivial subgroup of $Z(Q)$, completes the proof of the lemma.

Lemma 8. $G=F_{3}(G)$.

Proof. Suppose by way of contradiction that $G>F_{3}(G)$. It follows from [8] that $G_{\omega} \leqq F_{3}(G)$. Therefore $\omega$ induces a regular automorphism on $G / F_{3}$ so that $G / F_{3}$ is abelian. If $H$ is any subgroup of $G$ containing $F_{3}$ then by $\S 2$ lemma 3, corollary 2 , since $G_{\omega}<F_{3} \leqq H, H^{\omega}=H$. Since $G / F_{3}$ is abelian, $H$ is a normal subgroup of $G$. Suppose that $H \neq G$. Then $H$ satisfies the hypothesis of the theorem and therefore, by the minimality of $G$, $H=F_{3}(H)$. Since $H$ is normal in $G, F_{3}(H) \leqq F_{3}$ so $F_{3}=F_{3}(H)=H$. It follows that $G / F_{3}$ is cyclic of prime order.

Since $G_{\omega}<F_{3}<G, M_{\omega} \leqq F_{2}(M)<M$ and by $\S 2$, lemma 3, we can choose an element $x \in M$ such that $M=\left\{x, F_{2}(M)\right\}$ and $x^{\omega}=x^{-1}$. Now consider the $\omega$-subgroup of $G, K=\left\{x, Q, F_{1}\right\}$. Since $x^{\omega}=x^{-1}$, whilst $F_{2}=Q F_{1}$ is a normal subgroup of $K, K_{\omega} \leqq\left(Q F_{1}\right)_{\omega}$. But $Q$ is an abelian $p^{\prime}$-group, $F_{1}$ is an abelian p-group, and $G_{\omega}$ is nilpotent; therefore $K_{\omega}$ is abelian. Thus, as the theorem is true for $r=1, K^{\prime}$ is nilpotent.

Write $K^{\prime}=A \times B$ where $A$ is a Sylow $p$-subgroup of $K^{\prime}$. Then $B$ is a normal $p^{\prime}$-subgroup of $K$ and since $F_{1}$ is a $p$-group, $B \cap F_{1}=1$. Since $F_{1}$ is also a normal subgroup of $K$ and $G$ is soluble,

$$
B \leqq C_{K}\left(F_{1}\right) \leqq C_{G}\left(F_{1}\right) \leqq F_{1} .
$$

Thus $B=1$ and therefore $K^{\prime} \leqq Q F_{1}$ is a $p$-group. Therefore $K^{\prime} \leqq F_{1}$. Let $L=\{x, Q\}$. Then $L$ is a subgroup of $M$ and $K=F_{1} L$. Now $L \cong K / F_{1}$ is abelian so that $x \in C_{M}(Q)$. But $Q=F(M)$ and $M$ is soluble, so this implies that $x \in Q$. This contradiction to the choice of $x$ proves the lemma.

Corollary. Since $G=F_{3}(G)$ by lemma 8 , whilst $G$ does not satisfy the conclusion of theorem 1 , it follows that $G^{(r)}$ is not nilpotent. Thus $M^{(r)}>1$. 
LEMMA 9. There exists an $\omega$-complement $D$ of $Q$ in $M . Q$ is a q-group for some prime $q \neq p$ and $M / Q$ is a $q^{\prime}$-group.

Proof. To construct an $\omega$-complement of $Q$ in $M$ we use properties of Sylow systems of a soluble group (see [5] and [6]).

Since $M$ is soluble there exists a Sylow system of $M$. Since all such Sylow systems are conjugate in $M$ and since the order of $M$ is odd, there is an odd number of Sylow systems of $M$. The automorphism $\omega$ maps any given Sylow system of $M$ onto another and since $\omega$ has order 2, at least one Sylow system of $M$ is fixed by $\omega$. Form the system normalizer $D$ of this system. Clearly $D$ is an $\omega$-group and by the covering theorem, since $M=F_{2}(M), M=D Q$. Suppose that $D \cap Q>1$. Then since $Q$ is a normal subgroup of $M, D \cap Q$ is a normal subgroup of $D$. Also $Q$ is abelian, so that $D \cap Q$ is a normal subgroup of $D Q=M$. Let $K$ be a minimal normal subgroup of $M$ contained in $D \cap Q$. Then by the covering theorem, since $K \leqq D, K$ is centralized by $M$. But by the remark at the end of the proof of lemma $6, Z(M)=1$. Hence $K=1$ and therefore $D \cap Q=1$. Thus $D$ is an $\omega$-complement of $Q$ in $M$.

We next show that if $K$ is a proper $\omega$-subgroup of $M, K^{(r)}=1$. For if $K$ is a proper $\omega$-subgroup of $M, F_{1} K$ is a proper $\omega$-subgroup of $G$. Since $\left(G_{\omega}\right)^{(r)}=1,\left(\left(F_{1} K\right)_{\omega}\right)^{(r)}=1$, and therefore the minimality of $G$ implies that $\left(F_{1} K\right)^{(r)}$ is nilpotent. Thus we may write $\left(F_{1} K\right)^{(r)}=A \times B$ where $A$ is a $p$-group and $B$ is a $p^{\prime}$-group. By the minimality of $G, M^{(r)} \leqq Q$ so $\left(F_{1} K\right)^{(r)} \leqq F_{1} Q$. Thus $A \leqq F$. Also $B \triangleleft F_{1} K, F_{1} \triangleleft F_{1} K$ and as their orders are relatively prime, $B \cap F_{1}=1$. Hence $B \leqq C_{G}\left(F_{1}\right)=F_{1}$ so $B=1$. Now $\left(F_{1} K\right)^{(r)}=A \leqq F_{1}$ so $K^{(r)} \leqq F_{1} \cap M=1$ as required.

Now suppose that $Q$ is not a $q$-group for any prime $q$. Then we may write $Q=Q_{1} Q_{2}$ where $Q_{1}$ and $Q_{2}$ are Hall subgroups of $Q$ of relatively prime orders. Since $Q=F(M)$, the $Q_{i}$ are normal $\omega$-subgroups of $M$. Thus for each $i, D Q_{i}$ is a proper $\omega$-subgroup of $M$ and so $\left(D Q_{i}\right)^{(r)}=1$. Since $Q_{i}$ is abelian, it follows that

$$
\left(Q_{i}, D, D^{\prime}, \cdots, D^{(r-1)}\right)=1 \quad(i=1,2) .
$$

Also $D$ is a proper $\omega$-subgroup of $G$ sq that $D^{(r)}=1$. Now

$$
\begin{aligned}
M^{(r)} & =\left(D Q_{1} Q_{2}\right)^{(r)} \\
& =D^{(r)}\left(Q_{1}, D, D^{\prime}, \cdots, D^{(r-1)}\right)\left(Q_{2}, D, D^{\prime}, \cdots, D^{(r-1)}\right) \\
& =1,
\end{aligned}
$$

using, in addition to the above results, the fact that $Q=Q_{1} Q_{2}$ is an abelian group. But this contradicts the corollary to lemma 8 . Thus $Q$ is a $q$-group for some prime $q \neq p$. 
Since $M / Q$ is nilpotent, $Q$ is a $q$-group and $Q=F(M)$ it follows that $D \cong M / Q$ is a $q^{\prime}$-group.

LEMMA 10 . $D_{\omega}=D$.

Proof. Suppose that $D_{\omega}<D$. Then, since $D \cong M / Q$ is nilpotent by lemma, 8, there exists a proper normal subgroup $K$ of $D$ containing $D_{\omega}$. Form $K Q F_{1}$, a proper normal subgroup of $G$. Since $G_{\omega}=\left(F_{1}\right)_{\omega} Q_{\omega} D_{\omega}$ is contained in $K Q F_{1}, K Q F_{1}$ is an $\omega$-subgroup of $G$ by $\S 2$, lemma 3 , corollary 2. Hence by the minimality of $G,\left(K Q F_{1}\right)^{(r)} \leqq F_{1}$ and therefore $\left(K Q F_{1}\right)^{(r-1)}$ $\leqq F_{2}=F_{1} Q$. Thus $D_{\omega}^{(r-1)} \leqq K^{(r-1)} \leqq D \cap F_{1} Q=1$. Since $r>1$, $G_{\omega}$ is nilpotent and $D$ is a $q^{\prime}$-group whilst $Q$ is a $q$-group, $M_{\omega}=D_{\omega} Q_{\omega}$ has derived length at most $r-1$. Thus $M^{(r-1)} \leqq F(M)=Q$ and since $Q$ is abelian $M^{(r)}=1$. But this contradicts the corollary to lemma 8. Thus $D_{\omega}=D$.

Finally since $Q$ is abelian, $Q \leqq N_{M}\left(Q_{\omega}\right)$ and since $Q_{\omega}=Q \cap M_{\omega}$ and $D=D_{\omega} \leqq M_{\omega}, D \leqq N_{M}\left(Q_{\omega}\right)$. Thus $Q_{\omega} \triangleleft D Q=M$ contradicting lemma 6 . This contradiction completes the proof of the theorem.

\section{Proof of theorem 2}

Suppose that the theorem is false and choose a counterexample $G$ of minimal order. Then $F(G)$ is the unique minimal normal $A$-subgroup of G. $F(G)$ is an elementary abelian $p$-group for some prime $p$.

Let $\Gamma$ denote the splitting extension of $G$ by $A$ and write $F=F(G)$.

Suppose that $(G / F)_{\omega}=G / F$ for some $\omega \in A, \omega \neq 1$. Then since $G_{\omega}$ is nilpotent, $G / F$ is nilpotent. It is now an easy consequence of the minimality of $G$ that $G / F$ is a $q$-group for some prime $q \neq p$. Therefore we can choose a Sylow $q$-subgroup $Q$ to complement $F$ in $G$. Since $N_{\Gamma}(Q) F=\Gamma$, by taking a suitable conjugate of $Q$ if necessary, we may assume $A$ normalizes $Q$. Since $(G / F)_{\omega}=G / F, Q_{\omega}=Q$. Now $Z(G)=1$ for if $Z(G)>1 . Z(G) \geqq F$ which is false since $G$ is soluble. Since $G_{\omega}$ is nilpotent and $Q=Q_{\omega}$ is a group of order prime to $p$, whilst $F$ is an abelian $p$-group,

$$
F_{\omega}=G_{\omega} \cap F \leqq Z(F Q)=Z(G)=1 .
$$

Therefore $F_{\omega}=1$. Now we may write $\omega=\omega_{1} \omega_{2}$ where $\omega_{1}$ and $\omega_{2}$ are non-trivial elements of $A$. Since $Q_{\omega_{1} \omega_{2}}=Q$, it follows that $Q_{\omega_{1}}=Q_{\omega_{2}}$. Now form $F_{\omega_{1}}$ and $F_{\omega_{2}}$. Since $F_{\omega_{1} \omega_{2}}=1$, it follows from $\S 2$, lemma 3, that $F=F_{\omega_{1}} F_{\omega_{2}}$. Now $G_{\omega_{1}}$ and $G_{\omega_{3}}$ are nilpotent so, as before, $Q_{\omega_{1}}=Q_{\omega_{3}}$ is centralized by $F_{\omega_{1}}$ and $F_{\omega_{2}}$. Therefore $Q_{\omega_{1}} \leqq C_{G}\left(F_{\omega_{1}} F_{\omega_{2}}\right)=C_{G}(F)=F$. Thus $\omega_{1}$ induces a regular automorphism on $Q$, which implies that $Q$ is abelian. Since $G=F Q$, we conclude that $G^{\prime} \leqq F$ contrary to the definition of $G$. Therefore for no $\omega \in A, \omega \neq 1$, is $(G / F)_{\omega}=G / F$. 
If $F_{\omega}=1$ or $F$ for some $\omega \in A, \omega \neq 1$, then, as in $\S 3$, lemma 2 , $\omega$ either inverts or fixes all the elements of $F$ so that $(G / F)_{\omega}=G / F$. Since we have already shown that this is false, we conclude that for each $\omega \in A, \omega \neq 1$, $F>F_{\omega}>1$. Also, since $C_{G}(F)=F, C_{\Gamma}(F)=F$.

We have shown that $C_{\Gamma}(F)=F$ so it follows that $F=F(\Gamma)$ is the unique minimal normal subgroup of $\Gamma$. Therefore we may deduce (see [1]) that there exists a complement $N$ of $F$ in $\Gamma$. By Sylow's theorem we may suppose that $A \leqq N$. Let $M=G \cap N$ and $F(M)=Q$. The modular law implies that $M$ is a complement of $F$ in $G$.

For convenience we now summarize the properties of $F$ which we have obtained.

(a) $F$ is the unique minimal normal subgroup of $\Gamma$.

(b) $C_{\Gamma}(F)=F$.

(c) If $\omega \in A, \omega \neq 1$ then $Q_{\omega} \leqq C_{G}\left(F_{\omega}\right)$. This follows since $F$ is a $p$-group, $Q \cong F_{2}(G) / F$ and $G_{\omega}$ is nilpotent.

(d) For each $\omega \in A, \omega \neq 1, F_{\omega}>1$.

Properties (a) and (b) enable us to consider $F$ as a faithful irreducible $\Gamma / F$-module over $G F(p)$. Applying the same method as in the proof of $\S 3$, theorem 1, we may deduce the existence of an $\mathscr{F}(N)$-module $V$, where $\mathscr{F}$ denotes the algebraic closure of $G F(p)$, with the following properties:

(1) $V$ is a faithful irreducible $N$-module over $\mathscr{F}$.

(2) For each $\omega \in A, \omega \neq 1, V \omega=\{v \in V \mid v \omega=v\}>0$.

(3) For each $\omega \in A, \omega \neq 1$, if $v \in V_{\omega}$ and $x \in Q_{\omega}$ then $v x=v$.

We now show

(i) $V$ is an irreducible $\mathscr{F}(M)$-module.

Suppose, by way of contradiction, that $V$ is not an irreducible $\mathscr{F}(M)$ module. Let $W$ be an irreducible $\mathscr{F}(M)$-submodule of $V$. Then for at least two elements $\omega_{1}, \omega_{2} \in A,\left(\omega_{1}, \omega_{2} \neq 1\right)$ we have $W \omega_{1} \neq W$ and $W \omega_{2} \neq W$. Let $w \in W$ so that $w+w \omega_{i} \in V_{\omega_{i}}(i=1,2)$. Now if $y \in Q_{\omega_{i}}$ then by property (3)

$$
\left(w+w \omega_{i}\right) y=w+w \omega_{i} .
$$

Equating the $W$ components of each side, we deduce that $Q_{\omega_{i}}$ acts trivially on $W$ and so on $V$. But $V$ is a faithful $N$-module over $\mathscr{F}$ so it follows that $Q_{\omega_{i}}=1$ for $i=1,2$. By $\S 2$, lemma 3, $\omega_{1}$ and $\omega_{2}$ both invert all the elements of $Q$ so that $\omega_{1} \omega_{2}$ fixes all the elements of $Q$. Now we have already shown that $F_{\omega_{1} \omega_{2}}>1$ so since $F$ is an abelian $p$-group, $Q$ is of order prime to $p$ and $G_{\omega_{1} \omega_{2}}$ is nilpotent, $F_{\omega_{1} \omega_{2}} \leqq Z\left(Q_{\omega_{1} \omega_{2}} F\right)=Z(Q F)=Z\left(F_{2}(G)\right)$. Therefore $Z\left(G_{2}(G)\right)>1$. But $Z\left(F_{2}(G)\right)$ is a normal $A$-subgroup of $G$ so as $F$ is the unique minimal normal $A$-subgroup of $G, F \leqq Z\left(F_{2}(G)\right)$. This implies that 
$F_{2}(G)$ is nilpotent, a contradiction since $G$ is soluble and non-nilpotent. This contradiction proves (i).

In the same way as we proved $\S 3$ lemma 6 , we may now deduce

(ii) If $\omega \in A, \omega \neq 1$, and $L$ is a non-trivial normal $\omega$-subgroup of $M$, then $1<L_{\omega}<L$.

It follows from (ii), as in the remark after the proof of $\S 3$, lemma 6 , that

(iii) $Z(M)=1$.

This last result implies that $F_{2}(G)$ is a proper subgroup of $G$, so by the minimality of $G, Q \cong F_{2}(G) / F$ is abelian. We may also deduce from the minimality of $G$ that $M / Q$ is characteristically simple. Therefore $M / Q$ is an elementary abelian $r$-group for some prime $r$.

Suppose that $r$ divides the order of $Q$. Let $R$ be a Sylow $r$-subgroup of $M$. Since $Q$ is a normal subgroup of $M$ and $r$ divides the order of $Q$, $Z(R) \cap Q>1$. But $Z(R) \cap Q \leqq Z(R Q)=Z(M)$ since $Q$ is abelian. Thus $Z(M)>1$ contradicting (iii). We conclude therefore that $r$ does not divide the order of $Q$.

Let $R$ be a Sylow $r$-subgroup of $M$. Since $Q$ is of order prime to $r$, $R \cap Q=1$. Clearly $R Q=M$. Now form $N_{N}(R)$. It is easily shown that $N_{N}(R) Q=N$ so, by taking a conjugate of $R$ if necessary, we may suppose that $A$ normalizes $R$.

If $\omega \in A, \omega \neq 1$ is such that $R_{\omega}=R$, then since $M_{\omega} \leqq G_{\omega}$ is nilpotent, $r$ does not divide the order of $Q$ and $Q$ is abelian, $Q_{\omega} \leqq Z(R Q)=Z(M)$. Now on the one hand, (iii) implies that $Q_{\omega}=1$ whilst on the other hand(ii) implies $Q_{\omega}>1$, a contradiction. Thus for no $\omega \in A, \omega \neq 1$ is $R_{\omega}=R$.

It is an easy consequence of the minimality of $G$ that the representation of $A$ on $R$ is irreducible. But an irreducible representation, over a field of characteristic not equal to two, of the non-cyclic group of order 4 is onedimensional. Therefore for at least one $\omega \in A, \omega \neq 1$, is $R_{\omega}=R$ contradicting the conclusion of the last paragraph.

This contradiction completes the proof of theorem 2.

\section{Proof of theorem 3}

Proof. Suppose that the theorem is false and choose a counterexample $G$ of minimal order. Then $F=F(G)$ is the unique minimal normal $A$-subs group of $G$.

Let $L$ be a proper normal $A$-subgroup of $G$. Then $L$ is nilpotent. It follows that $F$ is the unique maximal normal $A$-subgroup of $G$ and that $G / F$ is an elementary abelian $r$-group for some prime $r$, since $G$ is soluble. Thus $G / F$ is an irreducible $A$-module over $G F(r)$. Now $A$ is of exponent two; so any irreducible representation of $A$, over a field of characteristic not equal 
to two, is one-dimensional. Therefore the kernel of the representation of $A$ on $G / F$ must have order 4 , at least. Let $\omega_{1}, \omega_{2}$ be two distinct non-unit elements of $A$ in the kernel. Then

$$
G / F=(G / F)_{\omega_{1}}=(G / F)_{\omega_{2}}=(G / F)_{\omega_{1} \omega_{\mathrm{s}}} .
$$

Suppose that $\omega \in A, \omega \neq 1$, and $(G / F)_{\omega}=G / F$. Since $F$ is the unique minimal normal $A$-subgroup of the soluble group $G, F$ is an elementary abelian $p$-group. By definition $G$ is not nilpotent, so $G / F$ is not a $p$-group. Therefore $r \neq p$. Now $(G / F)_{\omega}=G / F$ is isomorphic to a section of $G_{\omega}$ so the Sylow $r$-subgroup $R$ of $G_{\omega}$ is a complement of $F$ in $G$. Since $G_{\omega}$ is nilpotent and $F$ is abelian, $F_{\omega}=F \cap G_{\omega}$ is centralized by $R F=G$. Now if $Z(G)>1$, since $F$ is the unique minimal normal $A$-subgroup of $G, Z(G) \geqq F=F(G)$, a contradiction since $G$ is soluble. Therefore $F_{\omega} \geqq Z(G)=1$. It follows that $\omega$ inverts all the elements of $F$.

Combining these results we have for $x \in F$,

$$
x^{\omega_{1}}=x^{\omega_{2}}=x^{\omega_{1} \omega_{2}}=x^{-1} .
$$

Thus $x^{-1}=x^{\omega_{1} \omega_{2}}=\left(x^{-1}\right)^{\omega_{2}}=x$, a contradiction since the order of $F$ is odd. This proves the theorem.

\section{References}

[1] R. Baer, 'Nilpotent Characteristic Subgroups of Finite Groups', American J. Math. 75 (1953), $633-664$.

[2] C. Curtis and I. Reiner, Representation Theory of Finite Groups and Associative Algebras, Interscience, 1962.

[3] W. Gaschutz, 'Uber die $\Phi$-Untergruppe endlicher Gruppen', Math. Zeitschr., 58 (1953), $160-170$.

[4] M. Hall, Jr., The Theory of Groups, New York, 1959.

[5] P. Hall, 'On the Sylow Systems of a Soluble Group', Proc. London Math. Soc., (2) 43 (1937), 316-323.

[0] P. Hall, 'On the System Normalizers of a Soluble Group', Proc. London Math. Soc., (2) 43 (1937), 507-528.

[7] L. Kovács and G. E. Wall, 'Involutory Automorphisms of Groups of Odd Order and Their Fixed Point Groups', (to appear). Nagoya Math. $J$.

[8] J. Thompson, 'Automorphisms of Soluble Groups', J. Algebra 1 (1964), 259-267.

University of Sydney 